\title{
SELF-ORGANIZED CLUSTERING FOR FEATURE MAPPING IN LANGUAGE RECOGNITION
}

\author{
Chang Huai You, Kong Aik Lee, Bin Ma, Haizhou Li \\ Institute for Infocomm Research \\ Agency of Science, Technology and Research (A`STAR) \\ Fusionopolis, Singapore \\ \{echyou, kalee, mabin, hli\}@i2r.a-star.edu.sg
}

\begin{abstract}
In this paper, we propose a self-organized clustering method for feature mapping to compensate the channel variation in spoken language recognition. The self-organized clustering is realized by transforming the utterances into the Gaussian mixture model (GMM) supervectors and categorizing the supervectors through k-mean algorithm. Based on the language-dependent cluster-ofutterance information of the training databases, the feature mapping parameters are trained for each of the target languages. During recognition, the test utterance is identified to be one of the clusters according to the feature mapping parameters and then transformed into the cluster-independent features through feature mapping for a given target language. We show the effectiveness of the proposed self-organized feature mapping scheme through the 2003 National Institute of Standards and Technology (NIST) Language Recognition Evaluation (LRE) by using GMM recognizer.
\end{abstract}

\section{INTRODUCTION}

Automatic spoken language recognition is to recognize the language of an excerpt of speech uttered by an unknown speaker through training the language models. In the recognition system, the channel variation affects significantly the recognition accuracy. When a speech signal is recorded through a channel, it is corrupted by the channel related factor. When the speech signals for training and testing are from different channels, the mismatch between different channels results in a low recognition rate.

Compensation techniques for channel variation are generally applied in three domains: (a) feature domain compensation is to remove the channel effects from the feature vectors prior to model training or recognition [1]; (b) model domain compensation modifies the recognition models to minimize the effect of varying channels [2]; and (c) score domain compensation corrects the score scales and shifts caused by varying input channel conditions [3]. The feature domain compensation is the most general and widely used since it is independent from the particular recognition model or score configuration. The feature domain compensation includes the cepstral mean subtraction (CMS), RASTA processing [4], feature warping [5], feature mapping [6] and nonlinear filtering [7]. The CMS, RASTA and feature warping are a non-training-based compensation; and feature mapping and nonlinear filtering are training-based compensation.

Feature mapping technique has been effectively developed for speaker verification and speech recognition. It maps the channel-dependent feature into channel-independent features. In [6], Reynolds studies the feature mapping by categorizing the utterances based on channel information. This approach hinges on the availability of channel information. In [8], an iterative clustering method refines the context attribute of the training data to overcome inaccurate channel information. Based on the primary given channel-information of the training utterances, the feature mapping parameters are trained and obtained. With the trained feature mapping parameters, the training utterances is repeatedly reclassified in order to obtain more accurate context-attribute clusters.

In spoken language recognition, the training utterances are from a variety of languages. Usually, channel information is not easily available. In this case, the channel-based feature mapping may not be tractable. To address this issue, we propose a selforganized method to categorize the training utterances into different clusters. The aim is to seek the clusters with certain attributes from a set of training utterances by using self-organized clustering. Moreover, we introduce a feature mapping scheme for the language recognition, where the language-dependent mapping mode is proposed. The performance evaluation shows the effectiveness of the proposed self-organized feature mapping (SOFM) scheme for the language recognition system. In this paper, we focus on the feature-domain enhancement method by making use of the prior channel-like information. The remainder of the paper is organized as follows. In section 2, we describe our proposed self-organized clustering scheme and language-dependent feature mapping for language recognition. The performance evaluation is given in section 3, where we adopt the GMM recognizer to verify the validity of the self-organized clustering scheme through the NIST LRE-2003 test. Finally, section 4 summarizes the paper.

\section{PROPOSED SELF-ORGANIZED FEATURE MAPPING FOR LANGUAGE RECOGNITION}

In spoken language recognition, the training database involves a great amount of utterances in different languages. We seek to categorize the utterances based on a variety of speech attributes upon the utterances. It is fair to assume that every training utterance for language recognition is of unique language and spoken by unique speaker. Subsequently, it can be inferred that each utterance has its own range of attributes, such as nasal, fricative, speaking rate, duration of word-pause, pitch, recording channel, and speaking environment. The variation of these attributes lead to the bias from the universal language model which has been trained by using the utterances with a wide range of the attributes. In other words, a set of the utterances for training a language model can be categorized into many clusters in the space spanned by a range of the attributes.

We can separate the set of utterances into some clusters of the utterances by at least two ways. One way is to measure the values of the attributes of the utterances to form the attributive vectors and then separate them into different clusters in terms of the similarity of the vectors. However, there are some difficulties to estimate these values for the utterance. The problem on the accuracy and 
computational complexity may not allow the tractability. Another way is to blindly separate the utterances into a number of clusters through unsupervised-training parameters.

\subsection{Self-Organized Clustering}

In this paper, we propose a self-organized scheme to divide the utterances into different clusters, where the GMM supervector [9] is generated to represent the underlying attributes of the utterance. Like the channel-based cluster, the utterances within a self-organized cluster can be explained to be of certain similar underlying attributes. Different clusters have different ranges of underlying attributes. For each target language, each of the training utterances is transformed into a GMM supervector. Then the space spanned by the set of supervectors are divided into a number of subspaces through the k-mean method. Thus, the cluster-ofutterance information is obtained.

\subsubsection{GMM Supervectors for Self-Organized Clustering}

The probabilistic density of a feature vector $\mathbf{z}$ with the distribution of a GMM is given by

$$
p(\mathbf{z})=\sum_{i=1}^{\beta} w_{i} f\left(\mathbf{z} / \mathbf{m}_{i}, \Sigma_{i}\right)
$$

where $f(\cdot)$ denotes the Gaussian density, $\mathbf{m}_{i}$ and $\Sigma_{i}$ are respectively the mean and covariance of the $i$ th Gaussian component of the GMM, and $w_{i}$ is the weight of the Gaussian component.

First speech signal are transformed into the long term spectral feature, i.e., the shifted delta cepstral (SDC). We train a genderindependent global GMM universal background model (UBM) by using the utterances of all languages through Expectation Maximization (EM) algorithm. Second, using the global UBM as initial model, we train the gender-dependent GMM-UBM for each of the target languages through EM algorithm, which is referred to as language-dependent UBM. Lastly, the GMM of an utterance is obtained by adapting the features of the utterance through Maximum a Posteriori (MAP) algorithm from the language-dependent UBM. The GMM supervector is formed by concatenating its means which are normalized by standard deviations and weighted by the square root of the weight of Gaussian mixtures. i.e, each element of the GMM supervector is formed as follows

$$
\begin{gathered}
\varsigma((i-1) G+j)=\sqrt{w_{i}} \frac{m_{i}(j)}{\sigma_{i}(j)}, \\
j=1, \ldots, G ; \quad i=1, \ldots \beta
\end{gathered}
$$

where $G$ is the dimension of the feature vector, $m_{i}(j)$ is the $j$-th coefficient of $\mathbf{m}_{i}, \sigma_{i}(j)$ is the standard deviation equivalent to the square root of the $j$-th diagonal element of $\Sigma_{i}$ matrix, and $w_{i}$ is the weight of the $i$-th Gaussian component. In the GMM adaptation, only mean is adapted based on MAP, while weight and covariance are kept unchanged. In other words, $w_{i}$ and $\sigma_{i}(j)$ are the weight and standard deviation of the UBM respectively. Fig. 1 summarizes the process of generating the GMM supervector.

\subsubsection{Self-Organized Clustering by Using K-Mean Algorithm}

The underlying attributes represented by the supervectors are separated using the unsupervised clustering method. Self-organized implies a process that attempt to categorize the underlying characteristics from the given training utterances. There will be many approaches to categorize the supervectors. The purpose of this paper is to verify the validity of the self-organized approach but not

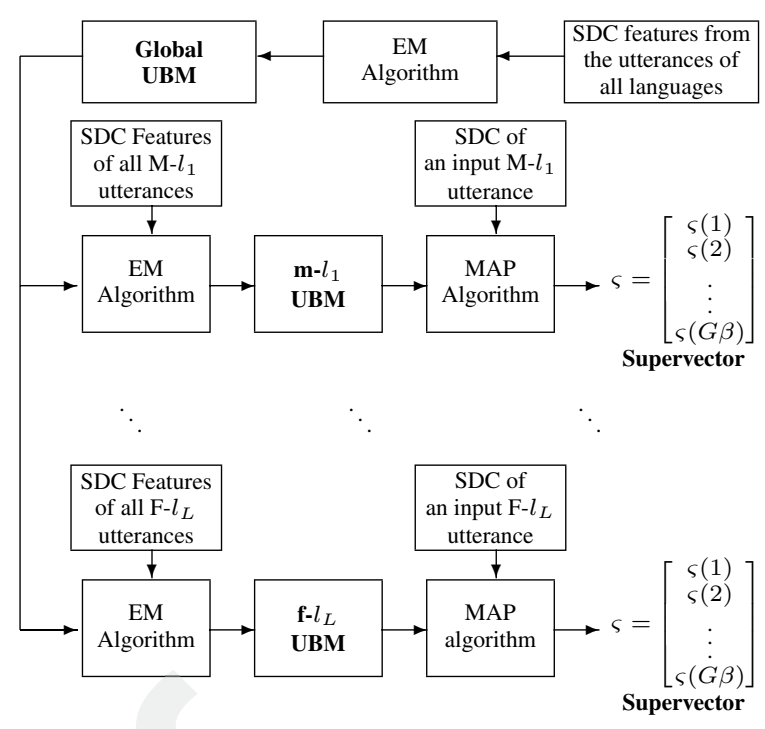

Fig. 1. The process of the generation of supervector of the utterance by using the training databases for the purpose of categorizing the utterances with underlying attributes. $\mathrm{m}-l_{1}$ and $\mathrm{f}-l_{L}$ denote the male language \#1 and female language \# $L$ respectively.

to study the approach to categorize the supervectors. For simplicity, we select to use k-mean algorithm [10]. The space spanned by the set of supervectors is divided into $M$ different subspaces. As a result, the set of utterances is divided into $M$ clusters of utterances with certain range of the values of the underlying attributes. The $\mathrm{k}$-mean algorithm works by repeatedly moving all cluster centers to the mean of their Voronoi sets. It iterates until no more cluster center is changed or the preset number of iterations reaches ${ }^{1}$.

In the self-organized clustering, if we use the languageindependent mode to do the self-organized clustering, an extreme situation is possible that some language has a whole amount of training utterances for a cluster while it has no training utterances assigned into other clusters. To avoid the unbalanced separation issue, we adopt the language-dependent clustering.

\subsection{Feature Mapping for the Language Recognition}

Similar to the feature mapping method in speaker verification system [6], which maps the feature vectors into a channel independent space, in this paper we apply the feature mapping method to map the features from the cluster-dependent space into the clusterindependent space.

\subsubsection{Training of Feature Mapping Parameters}

First a cluster-independent (CI) GMM with $N$-mixtures is trained using the utterances from all of clusters for each of the languages, which is referred to as a general GMM. For each language, a cluster-dependent (CD) GMM is obtained by adapting the training utterances belonging to a certain cluster from the general GMM by MAP. The SOFM can be viewed as an underlying attribute normalization by mapping the attribute-dependent feature space to the attribute-independent feature space. For each language, the feature mapping parameters are trained separately, where the training database of the language is used for the feature mapping parameter training. For every target language, there are $M$ GMMs to

${ }^{1}$ Usually, the initial cluster centers are randomly assigned. Therefore, slightly different results are possible. 
form the feature mapping parameters for each gender. Since there are $L$ target languages for each gender, we have $L$ set of feature mapping parameters. Therefore, there are total $2 L M N$ Gaussian components to describe the feature mapping parameters. Fig. 2 illustrates the procedure on training the feature mapping parameters. Although the training of feature mapping parameters is done for each of languages and therefore requires higher computational complexity, it is usually affordable since the computation is performed entirely in the parameter training phase without involving recognition phase.

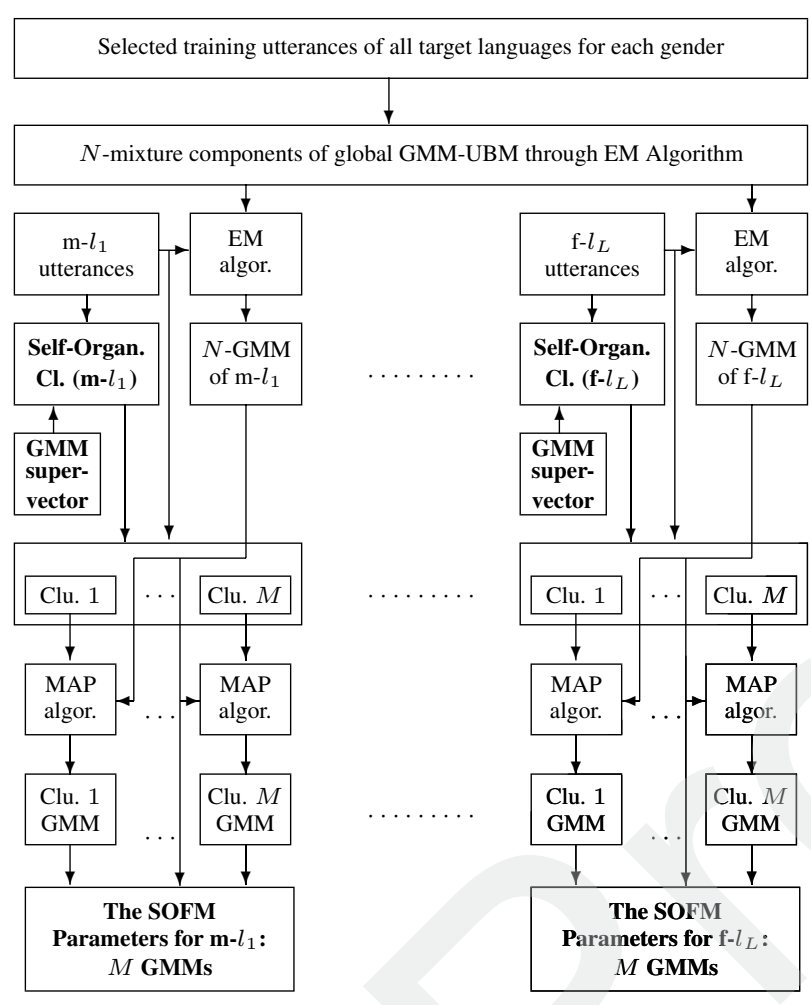

Fig. 2. The procedure of training the SOFM parameters for the language recognition.

\subsubsection{Feature Mapping During Recognition}

During recognition, given a testing utterance $\mathbf{Z}=$ $\left\{\mathbf{z}_{1}, \mathbf{z}_{2}, \cdots, \mathbf{z}_{T}\right\}$, the most likely cluster for a target language model is selected in the criterion of the maximum likelihood of utterance upon all of the cluster-dependent feature mapping GMMs of the target language $l$, i.e.,

$$
\breve{s}=\arg \max _{s \in[1, \ldots, M]}\left\{\sum_{k=1}^{N} w_{k} f\left(\mathbf{Z} / \mathbf{m}_{k}, \Sigma_{k}, \lambda^{(l)}(s)\right)\right\}
$$

where $s$ denotes the selected cluster to which the utterance belongs. For each feature vector, $\mathbf{z}_{t}$, in the utterance, the top Gaussian component of the selected cluster-dependent GMM $\lambda^{(l)}(s)$ is chosen among the $N$ Gaussian components,

$$
\breve{k}=\arg \max _{k \in[1, \ldots, N]}\left\{w_{k} f\left(\mathbf{z}_{\mathbf{t}} / \mathbf{m}_{k}, \Sigma_{k}, \lambda^{(l)}(\breve{s})\right)\right\} .
$$

Subsequently, the feature vector $\mathbf{z}_{t}$ is mapped to a clusterindependent space as follows

$$
\breve{\mathbf{z}}_{t}=\Sigma_{s i}^{(l)}(\breve{k})\left(\Sigma_{s d}^{(l)}(\breve{k})\right)^{-1}\left[\mathbf{z}_{t}-\mathbf{m}_{s d}^{(l)}(\breve{k})\right]+\mathbf{m}_{s i}^{(l)}(\breve{k})
$$

where $\Sigma_{s i}^{(l)}(\breve{k})^{T}$ and $\Sigma_{s d}^{(l)}(\breve{k})$ denote the respective covariance matrices of the $\breve{k}$ th cluster-independent and cluster-dependent Gausses for the target language $l$, while $\mathbf{m}_{s d}^{(l)}(\breve{k})$ and $\mathbf{m}_{s i}^{(l)}(\breve{k})$ are the corresponding mean vectors.

\subsection{The GMM Language Recognition System with SOFM}

We use $G$-dimension SDC coefficient [11] as the feature for the language recognition. The training databases are divided into the male and female groups. Consequently, the training for selforganized clustering, feature mapping parameter, and GMM language recognition are always gender-dependent in order to reach a higher accuracy of the recognition.

For the self-organized training, $G \beta$ dimension supervectors are generated for each of the gender-dependent target languages. Since $N$ mixtures of GMM is selected for the feature mapping parameter, we use $N$ mixtures for the GMM language recognition system. $\beta$-GMM is used to represent the utterance, it is trained by using the only-one utterance. On the other hand, $N$-GMM is to model the language, where there are many training utterances used for training. Due to the differences between the number of training SDC features, we select $\beta<<N$ to avoid the overfitting problem due to insufficient training features. i.e., the selection of the number of mixtures for GMM needs to keep the balance between the number of training features and the dimension of the trained parameters.

The SOFM method is ultimately applied in the recognition phase only. Given a language, the test utterance is processed by using the SOFM for the target language for both male and female respectively. Then score is obtained for the test utterances by averaging the logarithm of mixture Gaussian probability of all of the features for the target language model. For the language identification, one utterance has $L$ scores corresponding to the $L$ target language GMMs for both male and female respectively. We choose the male or female set of $L$ scores by comparing the maximum scores in the two sets.

\section{PERFORMANCE EVALUATION}

In this paper, we follow the experiment setup in the NIST LRE2003 task $^{2}$. The purpose of the LRE task is to build up a platform to measure the performance capability of the language recognition system on conversational telephone speech. In the LRE-2003 task, $L=12$ languages including Arabic, English, Farsi, French, German, Hindi, Japanese, Korean, Mandarin, Spanish, Tamil, and Vietnamese are involved. Three nominal durations of 3,10, and 30 seconds are assigned in the LRE task. For each duration there are 1280 test utterances.

In the evaluation, the 12-language LDC CallFriend database ${ }^{3}$ is adopted to train the parameters of the SOFM. The same database is used to train the GMM language recognizer. $\beta=10$ and 20 are selected for the self-organized clustering, and $N=512$ is chosen for the number of mixture components of the GMM for both feature mapping and GMM language recognition. The baseline feature is firstly extracted as follows: $12 \mathrm{Mel}$ frequency cepstral coefficients (MFCCs) are extracted, and the RASTA feature enhancement is applied. Then the MFCC is mapped into the shifted delta cepstral (SDC) feature with 7-1-3-7 pattern [12] [13], thus $G=49$. After SDC is produced, voice activity detection (VAD) and mean variance normalization (MVN) are applied.

It is possible, even we do not use the self-organized clustering scheme, we may reach an improvement by using random

\footnotetext{
${ }^{2}$ http://www.nist.gov/speech/tests/lang/2003/

${ }^{3} \mathrm{http}: / /$ www.ldc.upenn.edu
} 
Table 1. The comparison of the Equal Error Rates before and after the application of the SOFM with $\beta=10$ and 20 respectively by using the 512-mixtures of GMM on the LRE-2003 evaluation.

\begin{tabular}{|l|l|l|}
\hline GMM & \multicolumn{2}{|l|}{ EER (nominal duration: 30 sec.) } \\
\hline Score Processing & $T-N o r m$ & $L L N$ \\
\hline Baseline GMM & $7.15 \%$ & $7.23 \%$ \\
\hline RGFM GMM & $7.08 \%$ & $7.00 \%$ \\
\hline SOFM $\beta=10$ GMM & $6.67 \%$ & $6.60 \%$ \\
\hline SOFM $\beta=20$ GMM & $6.17 \%$ & $6.27 \%$ \\
\hline \hline GMM & \multicolumn{2}{|l|}{ EER (nominal duration: 10 sec.) } \\
\hline Score Processing & $T-N o r m$ & LLN \\
\hline Baseline GMM & $12.33 \%$ & $12.44 \%$ \\
\hline RGFM GMM & $11.89 \%$ & $12.38 \%$ \\
\hline SOFM $\beta=10$ GMM & $11.83 \%$ & $12.00 \%$ \\
\hline SOFM $\beta=20$ GMM & $11.61 \%$ & $11.25 \%$ \\
\hline \hline GMM & EER (nominal duration: 3 sec.) \\
\hline Score Processing & $T-N o r m$ & $L L N$ \\
\hline Baseline GMM & $22.37 \%$ & $22.52 \%$ \\
\hline RGFM GMM & $22.36 \%$ & $22.33 \%$ \\
\hline SOFM $\beta=10$ GMM & $22.15 \%$ & $22.50 \%$ \\
\hline SOFM $\beta=20$ GMM & $21.23 \%$ & $21.62 \%$ \\
\hline
\end{tabular}

clustering feature mapping as compared to the primary baseline GMM recognizer. In order to demonstrate the validity of the selforganized clustering, we have to compare the self-organized clusters with the random clusters which are formed by randomly selecting the utterances from the training database for each of the languages. Then, with the random clusters, we follow exactly the same feature mapping procedure as the SOFM. It is referred to random-generated feature mapping (RGFM). It will give a fair evaluation on the SOFM scheme by using the RGFM as a secondary baseline.

Table 1 gives the comparatively experimental results the language recognition system in terms of the equal error rate (EER) on the NIST LRE-2003, where the number of clusters is $M=2$ for both the RGFM and SOFM respectively. We apply the T-Norm and logarithmic likelihood normalization (LLN) on the scores of the GMM output. T-Norm is a mean and variance normalization over the 12 scores of a test utterance measured by the 12 target language GMM models. LLN is the logarithm likelihood normalization over the 12 scores of the test utterance.

From the experimental results, it is observed that (a) EERs with RGFM are very close to the baselines, it can only improve a very little for the GMM language recognition system; (b) the SOFM is consistently improve the GMM language recognition in terms of the EER, especially for the case of $\beta=20$; (c) the SOFM is of more effectiveness for the set of 30 seconds as compared to the set of 10 seconds, it becomes more apparent for the case of $\beta=10$. The phenomenon may be due to the difference of the durations between the training utterance and the testing utterance for SOFM. In this paper, we merely give very limited parametric settings for evaluation. Actually, the range of parametric settings such as $M, \beta$ and $N$ can be extended to a wider measure.

\section{SUMMARY}

In this paper, we introduced a self-organized clustering approach for feature mapping for language recognition. Against the lack of channel information, a self-organized clustering is an approach to realize the feature mapping. In the language recognition, we pro- posed to categorize the utterances by making use of GMM supervectors for every language. In a sequel, we proposed a languagedependent feature mapping scheme for the language recognition system. The SOFM is an unsupervised training scheme that develops a set of feature mapping parameters. It has been observed that the SOFM is of some effectiveness in the GMM recognition system through the 2003 NIST LRE.

\section{REFERENCES}

[1] L. Burget, P. Matějka, P. Schwarz, O. Glembek, and J. Černockỳ, "Analysis of feature extraction and channel compensation in a GMM speaker recognition system," IEEE Trans. on Audio, Speech and Language Processing, Vol. 15, No. 7, pp. 1979-1986, Sept. 2007

[2] R. Teunen, B. Shahshahani, and L. Heck, "A Model-based Transformational Approach to Robust Speaker Recognition,” ICSLP Oct. 2000.

[3] R. Auckenthaler, M. Carey, and H. Lloyd-Thomas, "Score Normalization for Text-Independent Speaker Verification SYstems," Digital Signal Processing, Vol. 10, pp. 42-54, Jan. 2000

[4] H. Hermansky and N. Morgan, RASTA processing of speech, IEEE Trans. Speech and Audio Processing, Vol. 2, No. 4, pp. 578-589, 1994.

[5] J. Pelecanos and S. Sridharan, "Feature warping for robust speaker verification," Proc. ISCA Workshop on Speaker Recognition - 2001: A Speaker Oddyssey, June 2001.

[6] D.A. Reynolds, "Channel Robust Speaker Verification via Feature Mapping," Proc. of Acoust., Speech \& Signal Process., ICASSP, Vol. 2, pp. 53-56, 2003.

[7] H. Jiang and Q. Wang, "Nonlinear noise compensation in feature domain for speech recognition with numerical methods," Proc. of Acoust., Speech \& Signal Process., ICASSP, Vol. 1, pp. 985-988, May 2004.

[8] M. Mason, R. Vogt, B. Baker and S. Sridharan, "Datadriven clustering for blind feature mapping in speaker verification," INTERSPEECH, pp. 3109-3112, 2005.

[9] W.M. Campbell, D.E. Sturim, and D.A. Reynolds, "Support Vector Machines Using GMM Supervectors for Speaker Verification," IEEE Signal Processing Letters, Vol. 13, pp. 308-311, 2006.

[10] T. Kanungo, D.M. Mount, N.S. Netanyahu, C.D. Piatko, R. Silverman, A.Y. Wu, "An Efficient k-Means Clustering Algorithm: Analysis and Implementation," IEEE Trans. on Pattern Analysis and Machine Intelligence, Vol. 24, No. 7, Jul. 2002.

[11] P. A Torres-Carrasquillo, E. Singer, M. A. Kohler, R. J. Greene, D. A. Reynolds, and J.R. Deller, Jr., "Approaches to Language Identification using Gaussian Mixture Models and Shifted Delta Cepstral Features," Proc. ICSLP, pp. 8992, 2002.

[12] W. M. Campbell, J.P. Campbell, D.A. Reynolds, E. Singer, and P.A. Torres-Carrasquillo, "Support vector machines for speaker and language recognition," Computer Speech and Language, No. 20, pp. 210-229, 2006.

[13] E. Singer, P.A. Torres-Carrasquillo, T.P. Gleason, W.M. Campbell, and D.A. Reynolds, "Acoustic, Phonetic, and Discriminative Approaches to Automatic Language Identification," Proc. Eurospeech, pp. 1345-1348, 2003. 